\title{
Identification of Precursory Anomalies before Moderate-strong Earthquakes in Junction of Shanxi-Hebei-Inner Mongolia Area
}

\author{
Li Hong $^{1}$ \\ ${ }^{1}$ Beijing Earthquake Agency, Beijing, China,100080
}

\begin{abstract}
In this paper, we take the Junction of Shanxi-Hebei-Inner Mongolia area as study region using earthquake corresponding relevancy spectrum method (ECRS method) to identify comprehensive precursory anomalies before moderate-strong earthquake. On base of single-parameter relevancy spectrum database with target earthquake magnitude as Ms4.7 and initial earthquake magnitude as Ms1, we carry on multi-parameter analysis and find that result with time interval of 9 months and anomaly threshold with 0.40 times standard deviation has better prediction efficiency. Its anomaly corresponding rate and earthquake corresponding rate are $6 / 10$ and $9 / 9$ respectively.
\end{abstract}

\section{Introduction and Method Brief}

The Junction of Shanxi-Hebei-Inner Mongolia is a historical earthquake-prone area located at the intersection of Zhangjiakou-Bohai tectonic belt and Fenwei earthquake belt. There are more than $20 \mathrm{NNE}$ right-lateral strike-slip and tensile tilt-slip faults. The area has been identified as a seismic hazard area for many years, so it is of great significance for the effective identification of seismic anomalies in this area. A lot of research indicate that different observed records and many seismic parameters deriving from earthquake catalogues may show different kinds of precursory anomaly before strong earthquakes. Many scholars have done lots of studies to explore different methods to extract and identify precursory anomaly before strong earthquakes based on mathematical statistics. Such as precursory recognition method of comprehensive information entropy [1], dynamic subordinate function method based on fuzzy mathematics[2], multipoint group slope and synthetic information flow method[3-4], normalized rate (slope) method[5]. Earthquake corresponding relevancy spectrum method (ECRS) was proposed by Wang Haitao et al. to identify comprehensive precursory anomaly before earthquakes and has been applied in many studies areas [6-14]. Here we will carry out precursory anomaly identification analysis with ECRS method.

The theory of ECRS method is as follows:

According to Wang Haitao et al.[6], for the observed time sequence $x_{1}, x_{2}, \ldots, x_{n}(i=1,2, \ldots, n), x_{\max }$ and $x_{\text {min }}$ are the maximum and minimum value, respectively. The mean and standard deviation can be obtained as follows:

$$
\begin{gathered}
\bar{x}=\frac{1}{n} \sum_{i=1}^{n} x_{i} \\
\sigma=\sqrt{\sum_{i-1}^{n}\left(x_{i}-\bar{x}\right)^{2} /(n-1)},
\end{gathered}
$$

On basis of formula (1) and (2), the value range distribution interval is defined as the following rule:

$$
\begin{aligned}
D x_{0} & \in(\bar{x}-k \sigma, \bar{x}+k \sigma) \\
D x_{1} & \in(\bar{x}+k \sigma, \bar{x}+2 k \sigma) \\
D x_{2} & \in(\bar{x}+2 k \sigma, \bar{x}+3 k \sigma), \\
D x_{n} & \in\left(\bar{x}+n k \sigma, x_{\max }\right), \\
D x_{-1} & \in(\bar{x}-2 k \sigma, \bar{x}-k \sigma), \\
D x_{-2} & \in(\bar{x}-3 k \sigma, \bar{x}-2 k \sigma), \\
D x_{-n} & \in\left(x_{\min }, \bar{x}-n k \sigma\right),
\end{aligned}
$$

According to formula (3) to (9), we can obtain range spectrum curve by counting the frequency of observed data sequence that distributed in the corresponding range. The value $k$ and $n$ can be modified so as to make the range spectrum curve more similar with the normal distribution as possible. If the data sequence is stable, $k$ should be small as 0.2 when data sequence is stable, $k$ should be big as 0.3 or so when it fluctuates. The value $n$ is between 6 and 10 usually.

Then we can calculate the earthquake corresponding relevancy spectrum (ECRS) in different time intervals by following steps. Firstly, determine target and initial earthquake magnitude according to seismic feature of study area. Secondly, count the number of observational 
sequence distributing in different interval of $D x_{-m}$ to $D x_{m}$ point-by-point and the number of earthquakes in different studied interval (unit: month). Then, count the data number corresponding with target earthquakes and all data number of corresponding range, and calculate the ratio of them (regarded as earthquake corresponding relevancy). So we can convert the time sequence of different seismic parameters $j(j=1,2, \cdots, k)$ into corresponding relevancy time sequence $P_{i j}$ ( $i=1,2, \cdots, n$ ) according to the affiliation value range point-by-point. Then we can obtain the sliding average correlation of different seismic parameters of time interval $t \quad(t=3,6,12,18)$ (unit: month) by formula $(10)$.

$$
\begin{gathered}
\bar{P}_{i j}=\left(p_{i j}+p_{(i+1) j}+\cdots+p_{(i+t-1) j}\right) / t, \\
i=1,2, \cdots,(n-t+1)
\end{gathered}
$$

We can get multi-point sliding extreme correlation sequence $\left(M_{i j}\right)$ on base of $\bar{P}_{i j}$ and the arithmetic average of $M_{j}$ of different parameter.

Set $j$ for different seismic parameters ( $j=1,2, \cdots, k), w$ for sliding window length (unit: month), then

$$
\begin{array}{r}
M_{i j}=\max \left\{\bar{P}_{i j}, \bar{P}_{(i+1) j}, ?, \bar{P}_{(i+w-1) j}\right\}, \\
i=1,2, \cdots, n-w+1 \\
M_{j}=\left(M_{1 j}, M_{2 j}, \cdots, M_{(n-w+1) j}\right), \quad j=1,2, \cdots, k
\end{array}
$$

Sliding extreme average correlation of multiparameters can be obtained as follow,

$$
P_{c}=\sum_{j=1}^{k} M_{j} / k
$$

\section{Data Preparation}

According to the geological structure and seismic activity characteristics of Junction of Shanxi-Hebei-Inner Mongolia Area, we determine the study area range is between $39.0^{\circ}-42.0^{\circ} \mathrm{N}$ and $111.5^{\circ}-116.0^{\circ} \mathrm{E}$ (see Fig.1). In this paper, we take small earthquake catalogue since January 1970 to March 2019 provided by China's Earthquake Network Center as data. Analysis of minimum magnitude completeness of the capital area by Wu minjie et al [15] shows that the minimum integrity magnitude of the whole area reached its highest value in 1986 and the minimum integrity magnitude can be controlled below ML2.0 since 1990. On base of ECRS method criteria, we select Ms4.7 as the target earthquake magnitude and Ms1 as initial earthquake magnitude.
Considering the accuracy and completeness of data, we delete aftershocks of earthquake catalogue by K-K method before time scan calculation. Here we calculate time sequence of seven seismic parameters such as $\eta$ value, $b$ value, seismic hazard $D$, earthquake $\operatorname{defect} \bar{M}$, seismic intensity factor $\mathrm{M}_{\mathrm{f}}$, seismic frequency $\mathrm{N}$ and seismic modulation ratio $\mathrm{R}_{\mathrm{m}}$, which are the original data for the further calculation.

Then, the earthquake corresponding relevancy spectrum database of each seismic parameter in Junction of Shanxi-Hebei-Mongolia area is established by using the ECRS method proposed by Wang Haitao et al. Here the studying database is from 1970 to 2017 and the extrapolating prediction period is from 2018 to March 2019. We can go to quantitative identification and extraction of comprehensive anomalies before moderate-strong earthquakes by retrospective earthquake example analysis.

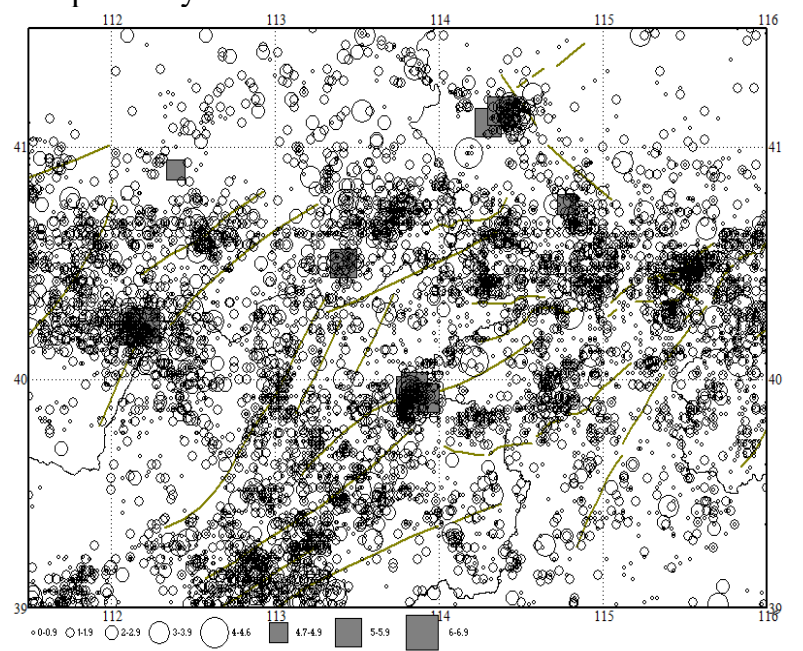

Fig.1 Earthquake ( $\geq \mathrm{Ms} 1)$ distribution of Junction of Shanxi-Hebei-Inner Mongolia Area since 1970 to March 2019 (square represent target earthquakes)

\section{Calculation results}

According to theory and criteria above, we get earthquake corresponding relevancy spectrum of each single parameter of Junction of Shanxi-Hebei-Inner Mongolia Area in different time intervals. And we calculate sliding extreme average correlation of multi-parameters on base of single-parameter results in corresponding time intervals. Due to the limitation of space, here we only give the final results of multi-parameters with time interval of 9 months, 12 months and 18 months (see Fig.2, Fig.3 and Fig.4). 


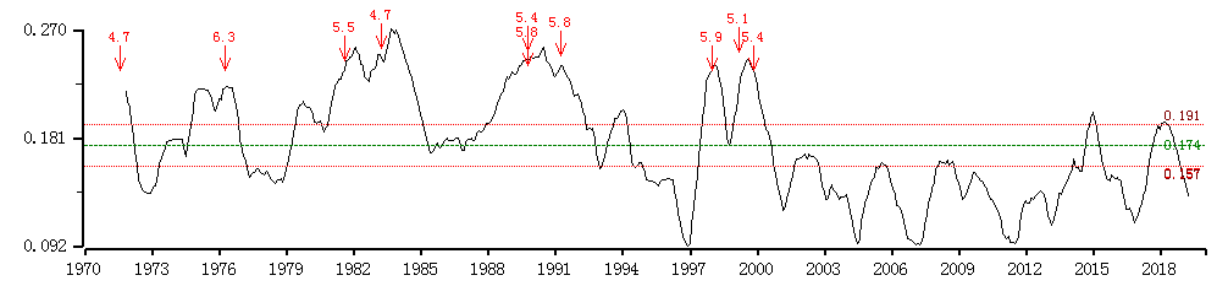

Fig.2 Multi-parameter sliding extreme average relevancy curve

of 9-month interval of Junction of Shanxi-Hebei-Inner Mongolia Area

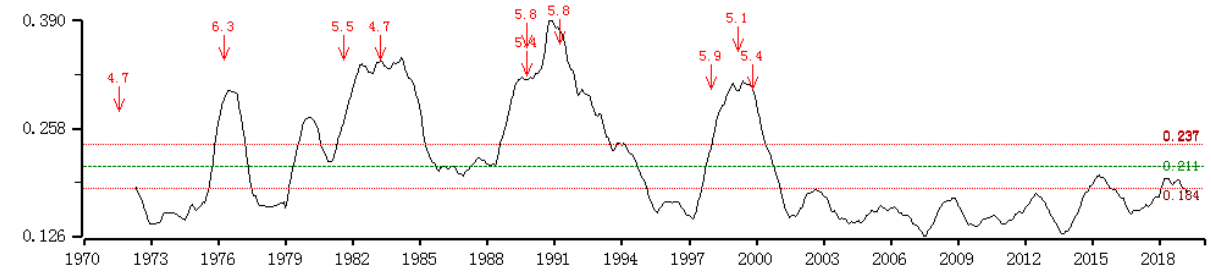

Fig. 3 Multi-parameter sliding extreme average relevancy curve

of 12-month interval of Junction of Shanxi-Hebei-Inner Mongolia Area

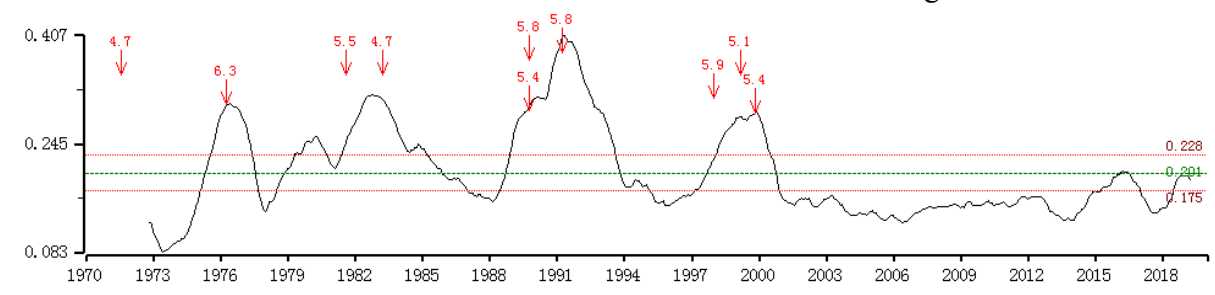

Fig. 4 Multi-parameter sliding extreme average relevancy curve

Of 18-month interval of Junction of Shanxi-Hebei-Inner Mongolia Area

During the calculation of above, We choose the proper parameter $k$ and $n$ by checking the range spectrum structure. And seismic parameters whose value range curve is more similar to the normal distribution were selected to participate in further calculation. We can see from Fig.2 to Fig.4, there appear high-value anomalies before most moderate-strong target earthquakes.

Here we take two indexes of anomaly corresponding proportion (number of anomalies corresponding to the target earthquake / total number of anomalies) and the earthquake corresponding proportion (number of target earthquakes with anomalies before the earthquake/ total target earthquakes) to describe the predictive efficiency of ECRS method. By comparing the results of different time intervals, we find the multi-parameters earthquake corresponding relevancy spectrum of 9-month has better predictive effectiveness than others with 0.40 times standard deviation as anomaly threshold. We regard the target earthquakes occurred in two months as a set, so there are 9 sets earthquakes and 10 high-value anomalies in total. The anomaly corresponding proportion and earthquake corresponding proportion of 9 time-interval are $6 / 10(60 \%)$ and $9 / 9(100 \%)$ respectively

\section{Conclusions and discussions}

Extraction and identification of precursory anomaly using ECRS method applied in Junction of Shanxi-Hebei-Inner Mongolia Area is the key work this paper. According to the analysis results, we can get some following conclusions.
Earthquake corresponding relevancy spectrum method is an effective identification method of earthquake precursory anomaly by retrospective case research and test. There are obvious high-value anomalies before most of the moderate-strong earthquakes in Junction of Shanxi-Hebei-Inner Mongolia Area, so we can carry out earthquake anomaly determination with ECRS method in study area.

Different seismic parameters could show different abnormal forms such as starting time, peak value, end time and so on before moderate-strong earthquakes. Multi-parameter corresponding relevancy spectrum shows more significant anomalies than that of single parameter. So in order to highlight the precursory anomalies of moderate-short period before earthquakes, we can calculate the multi-parameters earthquake corresponding relevancy spectrum on basis of single-parameter of that.

We use ECRS method to extract and identify precursory anomaly before moderate-strong earthquakes since 1970 to March 2019 of Junction of Shanxi-Hebei-Inner Mongolia Area with target earthquakes magnitude of Ms4.7. Different target earthquake magnitudes can lead to different results of predictive effectiveness.

Analysis results of different time intervals are a little different in precursory anomaly identification, so we should choose the better one by testing several different intervals. Study on Junction of Shanxi-Hebei-Inner Mongolia Area shows that multi-parameter earthquake corresponding relevancy spectrum with 9-month interval has higher prediction efficiency with anomaly corresponding proportion and earthquake corresponding 
proportion is $6 / 10$ and $9 / 9$ respectively. It indicates that the ECRS method can identify middle-short term comprehensive precursory anomalies in the study area.

Anomaly threshold is also a key factor to evaluate the predictive effectiveness. Here we take 0.45 times standard deviation as anomaly threshold, but it may be different from others researchers so that leads to different anomaly corresponding proportion and earthquake corresponding proportion.

Although we have achieved good precursory anomaly identification efficiency, there still have some misstatements and omissions and could be affected by subjective factors to some extent.

\section{Acknowledgements}

We appreciate the ECRS software provided by Wang Haitao, Wang Qiong and Tang Lanlan and anonymous reviewers. Thanks for the fund support of Three Integration Project (3JH-202001002), Earthquake Technology Spark Plan project (XH19001Y) and Earthquake Tracking project (2020010503).

\section{Author in Brief}

Li Hong, Ph. D. major in Tectonic Geology, senior engineer of Beijing Earthquake Agency, mainly engaged in seismic comprehensive analysis and geodynamics research.

\section{Reference}

1. Wang H.T, Zhu L.R, Yang M.L, et al. Analysis of information entropy of seismic precursory observation data-A case study of groundwater radon of well 10 in Urumqi.Earthquake,11(5):13-18. (1991)

2. Zheng X.M.,Feng D.Y.,Tian S. Study on dynamic subordinate function of earthquake precursory anomaly and its characteristic index $[\mathrm{J}]$.Earthquake, (1):36-43. (1994)

3. Bo W.J.,Wang Y., Luo S.M.. A new method of extracting anomalous information from monomial data-multipoint group information method [J] Earthquake Research in Shanxi, 1:39-42. (1996)

4. Bo W.J.,Wu Y.L.. Standardization Method and Application of Abnormal Information Flow [J]. Crustal Deformation and Earthquakes, 13(2):9-15. (1993)

5. Du X.B.,Ruan A.G.,Fan S.H.,et al. Anisotropy of the variation rate of apparent resistivity near the epicentral region of strong earthquakes[J].Acta Seismologica Sinica, 23(3):289-297. (2001)

6. Wang H.T.,Wang Q. Precursory Anomaly Identification Based on Earthquake Corresponding Probability Spectrum Analysis [J]. Journal of Seismological Research, 31(4):330-334(in Chinese). (2008)
7. Wang H.T., Wang Q., Tang L.L.. Comprehensive Precursory Anomaly Research Based on earthquake corresponding relevancy spectrum[J].Earthq Sci, 22(6):675-681. (2009)

8. Wang Q, Wang H.T., Tang L.L.. Research on Multi-parameter Comprehensive Anomaly Based on Earthquake Corresponding Probability Spectrum [J]. Earthquake Research in China, 25(3):294-302(in Chinese). (2009)

9. Wang Q.,Wang H.T.,Tang L.L.,et al. Multi-parametric anomaly spatial characteristics based on earthquake corresponding probability spectrum[J].Earthquake,30(1):98-107(in Chinese). (2010)

10. Tang L.L.,Wang H.T.,Wang Q. Research on anomaly of ECRS method before moderate-strong earthquakes in Xinjiang region[J].Northwestern Seismological Journal,33(2):159-165(in Chinese). (2011)

11. Feng J.G.,Wang Q, Tang L.L.. Research on precursory anomaly identification of the Gulang window earthquake based on ECRS method [J].Plateau Earthquake Research, 24(1):38-42(in Chinese). (2012)

12. LI H.,DENG Z.H.,XING Ch.Q.,et al. Application and test of ECRS earthquake anomaly analysis method in Beijing Area[J]. South china journal of seismology,37(2):1-9. (2017)

13. Lu X., Wang Q., Liu J., et al. Temporal-spatial Characteristic Anomaly before and after Moderate-strong earthquakes on the Tanlu Fault Zone [J]. Earthquake,32(1):40-48.(in Chinese). (2012)

14. Wang X.,Wang Q.,Feng J.G.,et al. The application of ECRS methods in Hebei Province and around area[J].Seismological and Geomagnetic Observation and Reaearch, 33(3/4):1-9.(in Chinese). (2012)

15. Wu M.J.,Wu A.X.,Long F.,et al. Temporal-spatial distribution characteristics of minimum magnitude of completeness in the Capital region of China[J],Earthquake,33 (3):98-106. (2013) 